\title{
Supernatants From Human Osteosarcoma Cultured Cell Lines Induce Modifications in Growth and Differentiation of THP-1 Cells and Phosphoinositide- Specific Phospholipase C Enzymes
}

\author{
Martina Leopizzi ${ }^{1}$, Valeria Di Maio ${ }^{1}$, Carlo Della Rocea ${ }^{1}$, Vincenza Rita \\ Lo Vasco ${ }^{2, *}$ \\ 'Department of Medico-Surgical Sciences and Biotechnologies, "Sapienza" \\ University of Rome - Polo Pontino, Latina, Italy \\ ${ }^{2}$ Department of Biomedical, Metabolic and Neural Sciences, Section of Mor- \\ phology, University of Modena and Reggio Emilia, Modena Italy \\ *Corresponding author: Vincenza Rita Lo Vasco, Department of Biomedical, \\ Metabolic and Nervous Sciences, Largo dal Pozzo, 1 - Modena, Italy. Tel: \\ +390594114804; Fax: +390594224861; E-mail: ritalovasco@hotmail.it; \\ vincenzarita.lovasco@unimore.it
}

DOI: $10.30699 /$ mci.4.4.430

Submitted: 23 May 2020

Revised: 19 June 2020

Accepted: 11 July 2020

e-Published: 1 October 2020

\section{Keywords:}

Phosphatidylinositols

Signal Transduction

Tumor Microenvironment

Monocyte-Macrophage
Introduction: Introduction: Molecular components within the microenvironment act upon cell growth, survival/apoptosis, and proliferation. Immune system cells respond to molecules produced by the tumor and released in the surrounding microenvironment, such as cytokines, chemokines, and growth factors. This study aimed to identify the effects of tumor environment on monocyte-macrophage cell lineage.

Methods: We evaluated morphological and functional changes in THP-1 cells cultured in culture medium mixed with the culture supernatant of one of three different osteosarcoma (OS) cell lines, namely 143B, HS888, and MG-63. We analyzed the effect of supernatants from OS cell lines on morphology and growth of THP-1 cells, and mRNA expression of phosphoinositide-specific phospholipase C (PLC) enzymes. Results: in supernatants from each OS cell line we identified the presence of selected interleukin (IL), TNF $\alpha$, and GM-CSF. Each OS-derived supernatant differently modified the growth rate of THP-1 cells, depending on the cell line. OS supernatants greatly modified the expression panel of PLC enzymes expressed by THP-1 cells in the in vitro microenvironment. THP-1 cells differently express PLC enzymes, depending on the origin of the supernatant. The differences in PLCs' expression induced by OS supernatants resulted in a statistically significant difference in expression of PLCB1 and PLCG2 genes.

Conclusions: OS supernatants induce the differentiation of THP-1 cells into macrophages. THP-1 cells cultured in OS supernatants expressed different expression panels of PLC enzymes at the mRNA level. The expression panel of PLC enzymes differs during the differentiation of monocyte/macrophage lineage THP-1 cells.

\section{INTRODUCTION}

The environment where cells live influences their growth, proliferation, and survival. Knowledge of the molecules contained in the microenvironment, including those belonging to signal transduction systems allowing crosstalk among cells, might help to understand both physiological events and systemic development of diseases [1]. The possibility to modify the microenvironment 
might open promising perspectives in terms of targeted molecular therapy approaches. In vitro experiments indicated that modifications of the molecular and biochemical components within the microenvironment can interact and influence the behavior of both cells in the surrounding normal tissue and cells belonging to the immune system [1] Immune system cells behave differently depending on selected stimuli from signal transduction pathways acting in the microenvironment.

In the present article, we cultured three human osteosarcoma (OS) cell lines and obtained the supernatant culture medium. OS is considered the most common primary bone tumor in children and adolescents [2]. The behavior of OS differs depending on the tumor cell type, probably influenced by different signal transduction pathways [3]. We cultured THP-1 cells in a culture medium; containing supernatant from OS cell lines. We aimed to verify the effect of OS-related microenvironment upon the differentiation of THP-1 cells. THP-1 monocyte-like cell line was derived from the peripheral blood of a one-year-old boy affected with acute monocytic leukemia and was treated with phorbol 12-myristate 13-acetate (PMA) in in vitro condition [4]. THP-1 cells can differentiate into macrophage-like phenotype under appropriate stimuli [5] and behave similarly to the native monocyte-derived macrophages [6]. After incubation with Interferon- $\gamma$ (IFN- $\gamma$ ) and Lipopolysaccharide (LPS) or with interleukin (IL)-4 and IL-13, THP-1-derived macrophages can be polarized respectively into the M1 or M2 phenotype [7]. After treatment with recombinant human IL-4 (rhIL-4) and recombinant human granulocyte-macrophage colony-stimulating factor (rhGM-CSF), THP-1 cells can differentiate into immature dendritic cells. After treatment with rhIL-4, rhGM-CSF, recombinant human tumor necrosis factor- $\alpha$ (rhTNF $\alpha)$ and Ionomycin, THP1 cells can also differentiate into mature dendritic cells [8]. Therefore, THP-1 cells are currently used as in vitro model to study the differentiation process of monocytes and macrophages [4].

The role of calcium and triggering elements of many intra- and intercellular events were extensively analyzed in the monocyte-macrophage line [9]. Great interest was reserved for the network of signaling molecules contributing to the complex regulation of calcium metabolism, including the Phosphoinositide (PI) pathway. PIs, minority acidic phospholipids play an instructional role in cell membranes and provide a complex and crucial intracellular signaling system involved in a variety of cell functions [10]. Crucial events are related to the regulation of phosphatidylinositol 4,5-bisphosphate (PIP2), a minor phospholipid located in the inner half-membrane [10] which can be hydrolyzed by enzymes belonging to the PI-specific phospholipase C (PLC) family. Mammalian PLC enzymes are grouped into six subtypes based on the amino acid sequence, domain structure, and mechanism of recruitment: $\beta(1-4), \gamma(1-2), \delta(1,3,4), \varepsilon, \zeta$, and $\eta(1-2)[10]$. Literature data described the role of the PLC signal transduction in development and differentiation pathway [11-14]. The expression panel of PLCs is tissue-specific [10, 13-22]. Also, the sub-cellular localization of PLC enzymes differs depending on the isoform and varies under different conditions; especially inflammation $[10,19,22]$. The activation of quiescent cells with different stimuli induced variation of the PLCs expression [22-25], and abnormal expression of PLCs was described in nervous anomalies $[3,13,14,16,17,26-28]$ and tumors $[17,18,23,24,29]$. PLC inhibition [22, $23]$ or silencing $[20,21,30]$ can modify the growth and survival of selected tumors. Recently, PLCdependent signaling was described to be crucial for macrophage chemotaxis [31]. The expression of PLC enzymes varies in unpolarized (M0), $\mathrm{M} 1$, and M2 macrophages in quiescent versus inflammatory-induced stimulation, with a peculiar panel of expression for each cell type [12].

In the present article, we evaluated the morphological and functional changes occurred in THP-1 cells cultured in a modified culture medium, obtained by replacing part of the Roswell Park Memorial Institute culture medium (RPMI) with supernatant from cultures of one of three different OS cell lines, namely 143B, HS888, and MG-63 cells. Secondarily, we analyzed the effects of supernatants from OS cell lines on enzymes involved in the metabolism of calcium in THP-1 cells. We analyzed the basic expression panel of PLC enzymes at the mRNA level in THP-1 cells by RT-PCR. Then, we analyzed modifications and the possible relationship with the morphological 
and functional changes induced in THP-1 cells by partially replacing the culture medium with supernatants derived from OS cell lines.

\section{METHODS}

\section{Culture of Osteosarcoma Cell Lines}

OS cell lines 143B, HS888, and MG63 were separately cultured as previously described [17]. MG-63, 143B, and Hs888 cells were obtained from the American Type Culture Collection (ATCC, Rockville, MD, USA). OS cell lines were separately grown under sub-confluent or confluent conditions at $37^{\circ} \mathrm{C}$ with $5 \%$ of $\mathrm{CO} 2$ in Dulbecco's minimum essential medium (DMEM) (Sigma) supplemented with $10 \%$ fetal bovine serum (GIBCO), penicillin $(100 \mu \mathrm{g} / \mathrm{ml})$, streptomycin $(100 \mathrm{U} / \mathrm{ml})$, and sodium pyruvate. Cells grew for 48 hours and reached $40-$ $60 \%$ confluence. Then, culture supernatants from each OS cell line were separately collected and stored at $-20^{\circ} \mathrm{C}$ until used for cytokine/chemokine analysis or culture of THP-1 cells.

\section{Biochemical Analysis of Osteosarcoma Super- natants}

The cytokine/chemokine profiles of supernatants were analyzed; using the Human Inflammatory Cytokines and Chemokines Multi-Analyte ELISArray Kit (QIAGEN, Hilden, Germany), following the manufacturer's instructions. The analysis was conducted for the following cytokines and chemokines: IL-1 $\alpha$, IL-1 $\beta$, IL-2, IL-4, IL-6, IL-8, IL-10, IL-12, IL-17A, IFN, TNF $\alpha$, and GMCSF.

\section{THP-1 Cultures}

A total of 200,000/well THP-1 cells were seeded into plates contenting $2 \mathrm{ml}$ of RPMI (controls) or $0.5 \mathrm{ml}$ RPMI (25\%) plus $1,5 \mathrm{ml}$ of supernatants $(75 \%)$ obtained from 48 hours cultured 143B or HS888 or MG-63 cells reaching to a final volume of $2 \mathrm{ml} /$ well. The modified culture media would be indicated as RPMI-s143B, RPMI-sHS888, and RPMI-sMG63; respectively. The number of viable cells treated with OS supernatant, pretrypsin, and attached cells post-trypsin treatment, was evaluated after 24, 48, and 72 hours and normalized for the number of viable THP-1 control cells at corresponding time intervals. After 48 hours, non-attached cells and adherent cells were counted; using a Neubauer hemocytometer. Cell pellets were stored at $-20^{\circ} \mathrm{C}$ until being used for molecular biology. The morphology of THP-1 cells was observed by optic microscopy. All experiments were conducted in triplicate. To evaluate the ability of differently cultured THP-1 cells to react to infections or remove tumor debris in the environment, the phagocytic ability was evaluated; using trypan blue 1:2 in culture medium for 2 hours. Then, cells were fixed in phosphatebuffered formalin and Acid Phosphatase enzymatic assay (Abcam, UK) was conducted following the manufacturer's instructions. The results were observed by optic microscopy. Statistical analysis of the results was conducted; using the ANOVA test. Results were considered statistically significant when $\mathrm{P}<0.05$.

\section{PLC Analysis}

mRNA from THP-1 cells cultured in RPMI (controls), RPMI-s143B, RPMI-sHS888, and RPMI-sMG63 were separately extracted with the SV Total RNA Isolation System (Promega, Madison, WI, USA) according to manufacturer's instructions. The purity of the RNA was assessed; using a UV/visible spectrophotometer (SmartSpec 3000, Bio-Rad Laboratories, Hercules, California, USA). The procedure, repeated in triplicates, was previously described [3]. mRNA was retrotranscribed into cDNA; using High-Capacity cDNA Reverse Transcription Kit as previously described [3](Life Technologies, Foster City, CA, USA). Two $\mu \mathrm{g}$ of mRNA were incubated with the master mix containing $2 \mu \mathrm{l}$ of $10 \mathrm{X}$ Reverse Transcription Buffer, $0.8 \mu 1$ of $25 \mathrm{X}$ dNTPs $(100 \mathrm{mM}), 2 \mu \mathrm{l}$ of $10 \mathrm{X}$ random primers, $1 \mu \mathrm{l}$ of MultiScribe TM Reverse Transcriptase (50 U/ $\mu \mathrm{l}$ ), and $3.2 \mu \mathrm{l}$ of DNase-free water to reach a final volume of $20 \mu \mathrm{l}$. PCR was performed; using the primer pairs (Bio Basic Inc, Amherst, New York, USA) listed in Table 1. PLCZ was not exclusively analyzed in the sperm lineage [10]. Glyceraldehyde 3 phosphate dehydrogenase (GAPDH) gene (Bio Basic Inc, Amherst, New York, USA) was used as an internal control; using the following primer pair: forward 5' -CGAGATCCCTCCAAAATCAA-3' reverse 5'-GTCTTCTGGGTGGCAGTGAT-3'. The primers were specificity rescued in the NCBI database for homologies to other cDNAs. We mixed $0.2 \mu \mathrm{M}$ of both sense and antisense primers, $1-3 \mu 1$ 
(about $1 \mathrm{mg}$ ) of template cDNA, $0.2 \mathrm{mM}$ of dNTP mix, 2.5U of REDTaq Genomic DNA polymerase (Sigma-Aldrich), and 1X of reaction buffer, $\mathrm{MgCl} 2$ at a variable concentration (empirical determination by setting the experiment) to reach a final volume of $50 \mu \mathrm{l}$. The amplification was started with an initial denaturation step at $94^{\circ} \mathrm{C}$ for 2 ' and was followed by 35 cycles consisting of denaturation (30") at $94^{\circ} \mathrm{C}$, annealing (30") at the appropriate temperature for each primer pair

Table 1: Primers' Pairs for Polymerase Chain Reaction of PLC Genes ${ }^{a}$

\begin{tabular}{|c|c|c|}
\hline & ОМIM & Primers for PLC Genes \\
\hline PLCB1 & $* 607120$ & $\begin{array}{l}\text { f: 5'-AGCTCTCAGAACAAGCCTCCAACA-3' } \\
\text { r: 5'-ATCATCGTCGTCGTCACTTTCCGT-3' }\end{array}$ \\
\hline PLCB2 & *604114 & $\begin{array}{l}\text { f: 5'-AAGGTGAAGGCCTATCTGAGCCAA-3' } \\
\text { r: 5'-CTTGGCAAACTTCCCAAAGCGAGT-3', }\end{array}$ \\
\hline PLCB3 & $* 600230$ & $\begin{array}{l}\text { f: 5'-TATCTTCTTGGACCTGCTGACCGT-3' } \\
\text { r: 5'-TGTGCCCTCATCTGTAGTTGGCTT-3' }\end{array}$ \\
\hline PLCB4 & $* 600810$ & $\begin{array}{l}\text { f: 5'-GCACAGCACACAAAGGAATGGTCA-3' } \\
\text { r: 5'-CGCATTTCCTTGCTTTCCCTGTCA-3', }\end{array}$ \\
\hline PLCG1 & $* 172420$ & $\begin{array}{l}\text { f: 5'-TCTACCTGGAGGACCCTGTGAA-3', } \\
\text { r: 5'-CCAGAAAGAGAG CGTGTAGTCG-3' }\end{array}$ \\
\hline PLCG2 & *600220 & $\begin{array}{l}\text { f: 5'-AGTACATGCAGATGAATCACGC-3' } \\
\text { r: 5'-ACCTGAATCCTGATTTGACTGC-3' }\end{array}$ \\
\hline PLCD1 & *602142 & $\begin{array}{r}\text { f: 5'-CTGAGCGTGTGGTTCCAGC-3' } \\
\text { r: 5'-CAGGCCCTCGGACTGGT-3' }\end{array}$ \\
\hline PLCD3 & *608795 & $\begin{array}{l}\text { f: 5'-CCAGAACCACTCTCAGCATCCA-3' } \\
\text { r: 5'-GCCA TTGTTGAGCACGTAGTCAG-3', }\end{array}$ \\
\hline PLCD4 & *605939 & $\begin{array}{l}\text { f: 5'-AGACACGTCCCAGTCTGGAACC- 3' } \\
\text { r: 5'-CTGCTTCCTCTTCCTCATATTC- 3' }\end{array}$ \\
\hline PLCE & $* 608414$ & $\begin{array}{r}\text { f: 5'-GGGGCCACGGTCATCCAC-3', } \\
\text { r: 5'-GGGCCTTCATACCGTCCATCCTC-3' }\end{array}$ \\
\hline PLCH1 & *612835 & $\begin{array}{l}\text { f: 5'-CTTTGGTTCGGTTCCTTGTGTGG-3' } \\
\text { r: 5'-GGATGCTTCTGTCAGTCCTTCC-3' }\end{array}$ \\
\hline PLCH2 & *612836 & $\begin{array}{l}\text { f: 5'-AAACTGGCCTCCAAACACTGCCCGCCG-3' } \\
\text { r: 5'-GTCTTGTTGGAGATGCACGTGCCCCTTGC-3' }\end{array}$ \\
\hline
\end{tabular}

and extension (1') at $72^{\circ} \mathrm{C}$. The PCR products were analyzed by $1.5 \%$ TAE ethidium bromidestained agarose gel electrophoresis (Agarose Gel Unit, Bio-Rad Laboratories S.r.l., Segrate, IT). Gel documentation was verified with a PC-mediated CCD camera UVB lamp (VilberLourmat, Marne-la-Vallé France). DNA contamination was excluded; performing PCR cycles without RT (data not shown). The concentration of the amplified cDNA was measured with Agilent 2100 bioanalyzer; using the DNA 1000 LabChip kit (Agilent Technologies, Deutschland GmbH).

\section{RESULTS}

\section{Analysis of Osteosarcoma Supernatants}

IL-2, IL-6, IL8, TNF $\alpha$, and GM-CSF were identified in the supernatant of all OS cell lines. IL-1 $\alpha$, IL-1 $\beta$ was identified in the supernatant from the 143B cell line. IL-4, IL-10, IL-12, IL-17, and IFN $\gamma$ were not identified in any supernatant (Table 2).

\section{THP-1 Cells' Culture}

The growth of THP-1 control cells in RPMI progressively increased after 24 (28\%), 48 (130\%), and $72(324 \%)$ hours. Percentages are intended to compare the initial number of viable seeded cells $(200,000)$ and cells counted at each time interval (Table 3, Figure 1A). The statistical analysis of the results was performed with the ANOVA test and the $\mathrm{P}<0.05$ was considered to be significant. The supernatants differently modified the growth rate of THP-1 cells, depending on the considered OS cell line. In THP-1 cells cultured in RPMI-s143, the growth rate increased similarly to controls (25\%) after 24 hours, decreased after 48 hours, and increased after 72 hours (Table 3, Figure 1A). In THP-1 cells cultured in RPMI-sHS888, the growth rate increased less than controls (12.5\%)

\begin{tabular}{|c|c|c|c|c|c|c|c|c|c|c|c|c|}
\hline & IL1A & IL1B & IL2 & IL4 & IL6 & IL8 & IL10 & IL12 & IL17A & INFG & TNFA & GMCSF \\
\hline C- & 0 & 0 & 0 & 0 & 0 & 0 & 0 & 0 & 0 & 0 & 0 & 0 \\
\hline 143B & 0.64 & 0.01 & 0.01 & 0 & 0.08 & 0.97 & 0 & 0 & 0 & 0 & 0.01 & 0.78 \\
\hline MG63 & 0 & 0 & 0.014903 & 0 & 10.43846 & 1.223523 & 0 & 0 & 0 & 0 & 0.01075 & 0.516883 \\
\hline HS888 & 0 & 0 & 0.01 & 0 & 1.406481 & 1.151484 & 0 & 0 & 0 & 0 & 0.012 & 0.021 \\
\hline $\mathrm{C}+$ & 1 & 1 & 1 & 1 & 1 & 1 & 1 & 1 & 1 & 1 & 1 & 1 \\
\hline
\end{tabular}


after 24 hours, increased after 48 hours, and increased after 72 hours (Table 3, Figure 1A). In THP-1 cells cultured in RPMI-sMG63, the growth rate increased as compared with controls (49\%) after 24 and 48 hours and decreased after 72 hours for the next 48 hours. The overall number of live THP-1 cells was greater than time 0 (Table 3 , Figure 1A). After trypsin, the survival rates of THP-1 cells greatly differed, both in control cells and in THP-1 cells cultured in the culture medium containing OS supernatants (Table 3, Figure 1B). In control cultures, the survival of THP-1 cells decreased after $24(-86.75 \%)$, and 48 hours, but was almost doubled after 72 hours (Table 3, Figure 1B). In THP-1 cells cultured in RPMI-s143, the survival rate decreased less than controls $(-77.5 \%)$ after 24 hours, increased after 48 hours, and decreased after 72 hours (Table 3, Figure 1B). In THP-1 cells cultured in RPMI-sHS888, the survival rate decreased less than controls $(-54 \%)$ after 24 hours, increased after 48 hours, and did not change after 72 hours (Table 3, Figure 1B). In THP-1 cells cultured in RPMI-sMG63, after 24 hours the survival rate decreased less than controls $(-77.5 \%)$ and similarly to RPMI-s143B cultured cells, increased after 48 and 72 hours (Table 3, Figure 1B). The statistical analysis of the results is available in Table 4, right columns. Although the partial replacement of culture medium with OS supernatant affected the growth and survival rates of THP-1 cells, the statistical analysis of these results was significantly exclusively for the supernatant from the HS888 cell line (Table 3).

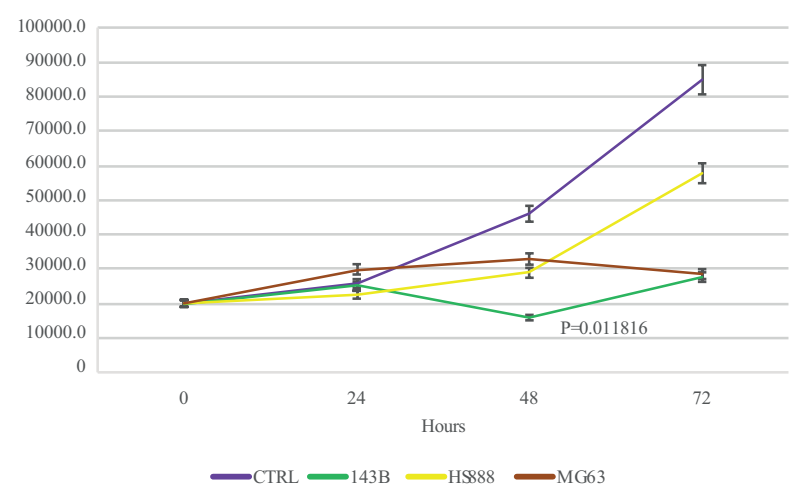

Figure 1: THP-1 Growth Curves

Graphic representations of the growth curve of THP-1 cells (CTRL), of THP-1 cells cultured in RPMI-143B supernatant (S143), of THP-1 cells cultured in RPMI-HS888 supernatant (SHS888), and THP-1 cells cultured in RPMI-MG63 supernatant (S-MG63). Standard error bars were indicated for each growth curve.

\begin{tabular}{|c|c|c|c|c|c|}
\hline & Seeding Time (T0) & $24, \mathrm{hr}$ & $48, \mathrm{hr}$ & $72, \mathrm{hr}$ & P Value \\
\hline \multicolumn{6}{|l|}{ Pre Trypsin } \\
\hline CTRL & 200,000 & 257,500 & 460,000 & 848,750 & 0.086 \\
\hline S-143 & 200,000 & 250,000 & 158,750 & 276,250 & 0.241 \\
\hline S-HS888 & 200,000 & 225,000 & 288,750 & 577,500 & 0.139 \\
\hline S- MG63 & 200,000 & 298,750 & 328,750 & 285,000 & 0.370 \\
\hline CTRL/s-143 & - & - & - & - & 0.086 \\
\hline CTRL/s-HS888 & - & - & - & - & 0.241 \\
\hline CTRL/s-MG63 & - & - & - & - & 0.139 \\
\hline CTRL/OOSS & - & - & - & - & 0.370 \\
\hline \multicolumn{6}{|l|}{ Post Trypsin } \\
\hline CTRL & - & 26,250 & 23,750 & 42,500 & 0.053 \\
\hline S-143 & - & 45,000 & 147,500 & 88,750 & 0.011 \\
\hline S-HS888 & - & 91,250 & 202,500 & 206,250 & 0.054 \\
\hline S-MG63 & - & 45,000 & 118,750 & 191,250 & 0.089 \\
\hline CTRL/s-143 & - & - & - & - & 0.053 \\
\hline CTRL/s-HS888 & - & - & - & - & 0.011 \\
\hline CTRL/s-MG63 & - & - & - & - & 0.054 \\
\hline CTRL/OOSS & - & - & - & - & 0.089 \\
\hline
\end{tabular}

${ }^{a}$ Abbreviations: CTRL, Viable Cells; S-HS888, THP-1 cells cultured in RPMI-HS888 supernatant; S-MG63, THP-1 cells cultured in RPMI-MG63 supernatant; S-143, THP-1 cells cultured in RPMI-143B supernatant

${ }^{\mathrm{b}}$ Pre trypsin: P values obtained comparing the number of cells and median of CTRL, S-143, S-HS888, and S-MG63. Post trypsin: P values obtained comparing the number of attached cells counted after trypsin treatment and median of CTRL, S-143, S-HS888, and S-MG63. 
Morphology Observations and Functional Assays

The trypan blue phagocytosis assays in THP-1 cells cultured in RPMI and each OS supernatant resulted positive; suggesting that THP-1 cells had acquired phagocytic capabilities (Figure 2). THP1 cells cultured in RPMI and each OS supernatant showed positive results to the Acid Phosphatase assay (Figure 3); suggesting possible differentiation into macrophages which were confirmed by the positivity to $\mathrm{CD} 68+$ immune-characterization (data not shown). THP-1 cells were adhered to culture plates, aggregated, elongated, and formed pseudopodia followed in a time-dependent manner (Figure 2, Figure 3). The ability of THP-1 cells cultured in supernatants from OS cell lines to phagocytize opsonized particles was enhanced compared to THP-1 control cells. The behavior of THP-1 cells cultured in the presence of OS supernatants resembled that of monocyte-derived macrophages for adherence and phagocytosis.

\begin{tabular}{|c|c|c|c|c|}
\hline & THP & M0 & M1 & M2 \\
\hline PLCB1 & + & $+/-$ & + & + \\
\hline PLCB2 & + & + & + & + \\
\hline PLCB3 & + & + & + & + \\
\hline PLCB4 & + & $+/-$ & - & - \\
\hline PLCG1 & + & + & + & + \\
\hline PLCG2 & + & + & + & + \\
\hline PLCD1 & + & + & + & - \\
\hline PLCD3 & + & - & + & + \\
\hline PLCD4 & + & - & - & - \\
\hline PLCE & + & - & - & - \\
\hline PLCH1 & + & + & + & + \\
\hline PLCH2 & + & + & + & + \\
\hline
\end{tabular}

\section{PLC Expression}

The mRNA of 12 PLC enzymes was detected in the THP-1 control cells. Cultured in RPMI-s143B, THP-1 cells did not express PLCD4 and PLCE at the mRNA levels (Table 5). Modifications were measured in the expression of the remaining isoenzymes (Table 5). These modifications resulted in a statistically significant difference for PLCB1and PLCG2 (Table 5, Figure 4). Cultured in RPMI-sHS88, THP-1 cells did not express PLCB1, PLCG2, PLCD4, PLCE, PLCH1, and
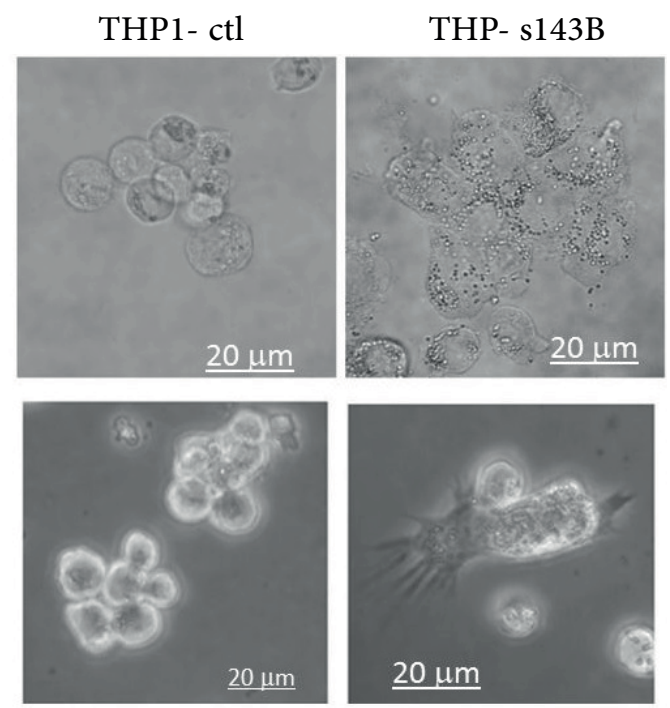

THP- sHs 888

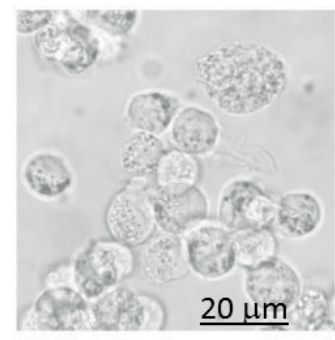

THP- sMG63
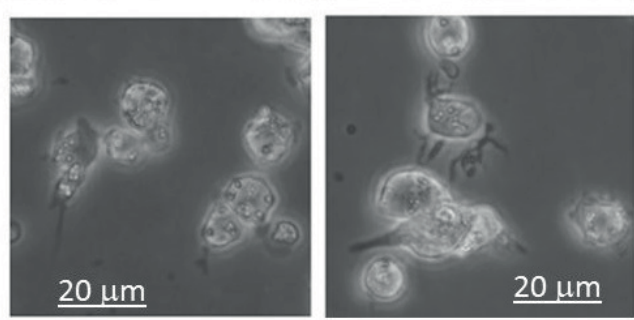

Figure 2: THP-1 Cells Morphology

Optic microscopy. THP-1 cells after 48 hours of culture. THP1 CTRL: controls grown in RPMI. THP-s143B: THP-1 cells grown in RPMI and supernatant from 143B cells. THP-Shs888: THP-1 cells grown in RPMI and supernatant from hs 888 cells. THP-Smg63: THP-1 cells grown in RPMI and supernatant from MG-63 cells (60X)

PLCH2 (Table 5, Figure 4). Modifications were measured in the mRNA expression of the remaining isoenzymes (Table 5). The variations of expression of PLCs resulted in a statistically significant difference between PLCB1 and PLCG2 (Table 5, Figure 4). Cultured in RPMI-sMG63, THP-1 cells did not express PLCB1, PLCD4, PLCE, PLCH1, and PLCH2. Modifications were measured in the expression of the remaining isoenzymes (Table 5, Figure 4). The modifications resulted in a statistically significant difference between PLCB1 and PLCG2 (Table 5). 


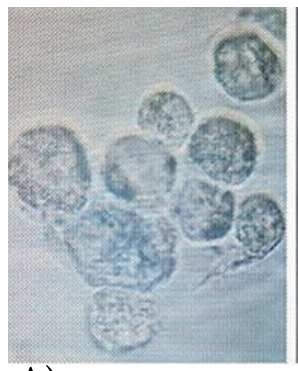

A)
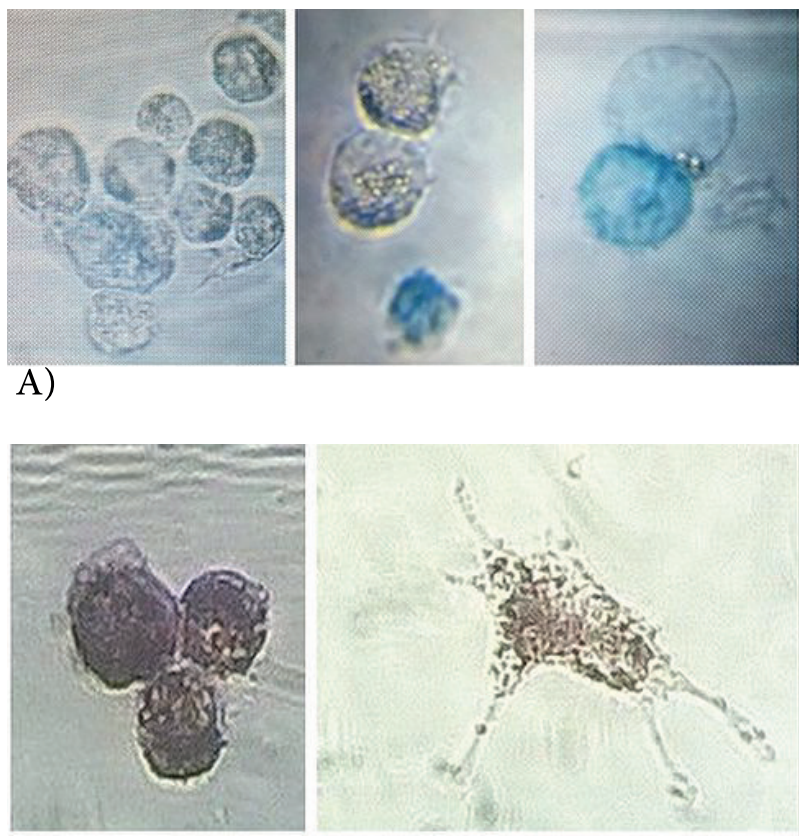

B)

Figure 3: THP-1 Functional Assays

A) Trypan blue phagocytosis assay after 2 hours. Positive cells (40X); B) Acid Phosphatase enzymatic assay. Positive cells $(60 X)$.

\section{DISCUSSIONS}

Tissue microenvironment is comprised of factors that directly or indirectly act upon cell behavior via biophysical, biochemical, or other routes [32]. In the present article, we cultured monocytemacrophage THP-1 cells with culture medium

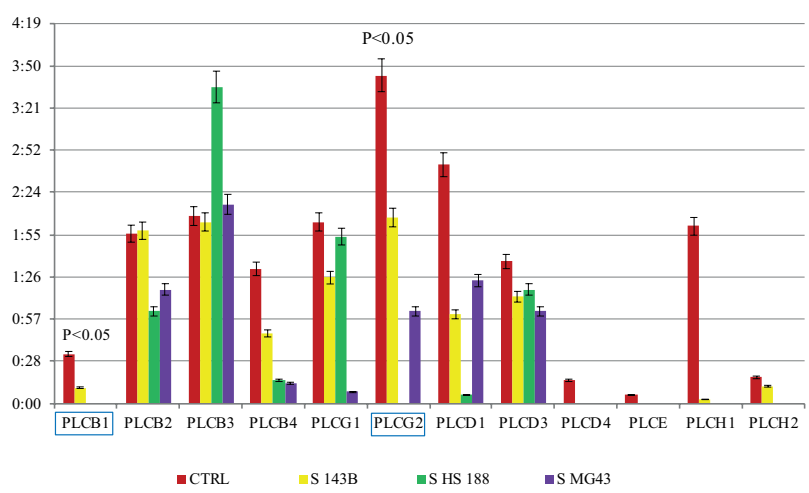

Figure 4: Graphical Representation of the Concentration of PLCs' mRNA in OS Cell Lines

Standard error bars were indicated for each PLC isoform. Isoforms in the box resulted in statistically significant.

containing supernatant derived from cultures of OS cell lines. We aimed to analyze whether factors released by OS cells within the supernatant could modify the morphology of THP-1 cells and the panel of expression at the mRNA level of PLC enzymes. Many pieces of evidence suggested that the microenvironment can affect the growth, proliferation, and survival of cells in the tissues. Further pieces of evidence suggested that under pathological conditions, additional/abnormal components in the microenvironment, produced as metabolites by pathological cells, might act upon related cells, i.e. inducing modifications in the morphological and functional features of immune system cells [33]. In vitro modifications of the microenvironment by partially replacing the

\begin{tabular}{|c|c|c|c|c|c|c|c|c|}
\hline & \multirow[b]{2}{*}{ CTRL } & \multirow[b]{2}{*}{ S-143B } & \multirow[b]{2}{*}{ S-HS888 } & \multirow[b]{2}{*}{ S-MG63 } & \multicolumn{4}{|c|}{ P Value ${ }^{\text {b }}$} \\
\hline & & & & & $\begin{array}{c}\text { CTRL/S- } \\
\text { 143B }\end{array}$ & $\begin{array}{c}\text { CTRL/S- } \\
\text { HS888 }\end{array}$ & $\begin{array}{c}\text { CTRL/S- } \\
\text { MG63 }\end{array}$ & $\begin{array}{c}\text { CTRL/All } \\
\text { Treat }\end{array}$ \\
\hline PLCB1 & 0.34 & 0.11 & 0 & 0 & 0.020 & $<0.001$ & $<0.001$ & 0.002 \\
\hline PLCB2 & 1.56 & 1.58 & 1.30 & 0.78 & 0.983 & 0.719 & 0.298 & 0.859 \\
\hline PLCB3 & 2.08 & 1.64 & 2.96 & 2.16 & 0.503 & 0.361 & 0.931 & 0.466 \\
\hline PLCB4 & 1.32 & 0.48 & 0.16 & 0.14 & 0.306 & 0.16 & 0.182 & 0.312 \\
\hline PLCG1 & 2.04 & 1.26 & 1.54 & 0.80 & 0.064 & 0.54 & 0.051 & 0.232 \\
\hline PLCG2 & 3.44 & 1.67 & 0 & 0.63 & 0.009 & 0.004 & 0.014 & $<0.001$ \\
\hline PLCD1 & 2.43 & 0.61 & 0.60 & 0.84 & 0.085 & 0.16 & 0.223 & 0.152 \\
\hline PLCD3 & 1.37 & 0.73 & 0.78 & 0.63 & 0.381 & 0.651 & 0.564 & 0.765 \\
\hline PLCD4 & 0.16 & 0 & 0 & 0 & 0.117 & 0.221 & 0.221 & 0.133 \\
\hline PLCE & 0.60 & 0 & 0 & 0 & 0.189 & 0.308 & 0.308 & 0.256 \\
\hline PLCH1 & 1.61 & 0.03 & 0 & 0 & 0.392 & 0.494 & 0.494 & 0.624 \\
\hline PLCH2 & 0.18 & 0.12 & 0 & 0 & 0.787 & 0.495 & 0.495 & 0.685 \\
\hline
\end{tabular}

a Abbreviations: CTRL, untreated THP cells; CTRL/All Treat, comparison of untreated THP and THP treated with overall treatments; S, supernatant from the OS cell line

${ }^{\mathrm{b}}$ Statistical analysis of PLCs' in controls compared to THP-1 cultured in OS supernatants. ANOVA test was used to compare concentrations of PLC genes' transcripts. 
culture medium with supernatants from different OS cell lines acted upon THP-1 cells. The results of our experiments slightly differed depending on the OS cell line. Partial replacement of culture medium with OS supernatants acted upon the survival and proliferation rate of THP-1 cells. The growth curves indicate the most relevant differences after 48 hours from seeding; suggesting that the effects of OS supernatants last more than one day. Although statistically not significant, modifications were also induced by RPMI-sHS888, which slowly reduced the growth of THP-1 cells for controls after 24 hours (Table 3, Figure 1A). Statistically not significant, modifications were also induced in THP-1 cells by RPMI-sMG63. The number of live and attached THP-1 cells cultured in RPMI and OS supernatant was greater than THP-1 control cells (Table 3, Figure 1); suggesting that THP1 cells grown in RPMI-OS supernatant become more resistant to trypsin digestion and detachment. Our results suggest that OS supernatants can modify the growth rate of THP-1 cells with the most significant effect played by the supernatant from the 143B cell line. That is not surprising, as 143B cells are known to be highly aggressive and migrating [34].

Microenvironment changes due to OS supernatants modified the morphological and functional features of THP-1 cells, inducing differentiation. The comparison of trypan blue phagocytosis assay in THP-1 cells cultured in RPMI and OS supernatant from each OS cell line demonstrated that THP-1 cells had acquired phagocytic capabilities (Figure 2). Similarly, cultured THP-1 cells also showed positive results to the Acid Phosphatase assay (Figure 3); suggesting their differentiation into macrophages, then confirmed by the positivity to $\mathrm{CD} 68+$ immune-characterization (data not shown). Morphology observations and functional tests indicated that THP-1 cultured with RPMIOS supernatant differentiated into cells behaving similarly to THP-1-derived macrophages. In this perspective, the cytokines and chemokines identified in the supernatants deserve consideration. The content of cytokine/chemokine in each OS supernatant differed; depending on the OS cell line (Table 2). In each OS cell line, we identified IL-2, IL-6, IL-8, TNF $\alpha$, and GM-CSF. IL- $1 \alpha$ and IL- $1 \beta$ were exclusively detected in the supernatant derived from cultures of 143B cells (Table 2). The differences among the cytokines contained in the three supernatants might be related to the different features of OS cells they were derived from. The MG-63 cell line is currently used as an experimental model for human osteoblasts [35]. The 143B cell line and HS 888 cell line derives from lung metastases of OS develop osteolytic tumors, sharing migration abilities and aggressiveness [36](Atcc.org). Notably, IL-2 is a central factor in controlling the immune response [37]. The remaining cytokines we identified in the supernatant of OS cell lines bear pro-inflammatory features [37]. GM-CSF and TNF $\alpha$ are known to induce the differentiation of THP-1 into dendritic cells [4, 8]. By contrast, IL-4, which can induce dendritic differentiation of macrophage polarisation, was not detected. Also, IL-17, known to bear regulatory role upon the activity of the immune system [38], was not detected in the supernatant from OS cell lines. The trypan blue phagocytosis assay suggested that THP-1 cells cultured in RPMI-OS supernatants had acquired phagocytic capabilities (Figure 2). Such THP-1 cells also showed positive results to the Acid Phosphatase assay (Figure 3); suggesting their differentiation into macrophages. This hypothesis was confirmed by the positivity to $\mathrm{CD} 68+$ immune-characterization (data not shown). THP-1 cells adhered to culture plates and aggregated. Elongation and formation of pseudopodia were detected in a time-dependent manner (Figure. 2, Figure 3) and the ability to phagocytize was enhanced compared to THP-1 control cells. Therefore, the behavior of THP-1 cells cultured in the presence of OS supernatants resembles that of monocyte-derived macrophages for adherence and phagocytosis. That might partially fit with the presence of cytokines such as GM-CSF, TNF $\alpha$, and IL-1 in the OS supernatants that are known to act upon monocyte-macrophage lineage differentiation $[37,39]$. The role of the cytokines in the OS microenvironment and their effect upon tumor growth and spreading will require further studies.

We analyzed the expression of PLC enzymes at the mRNA level which is known to play a role in macrophage differentiation [12]. All PLC isoforms were expressed in THP-1 cells that were grown in RPMI, except for PLC $\zeta$ which is 
exclusively present in the sperm lineage [10]. The presence of OS supernatants in the in vitro microenvironment greatly modified the mRNAs of PLC enzymes expressed by THP-1 cells (Table 1). Partial replacement of RPMI with supernatants from cultured OS cell lines, such as 143B, HS888, or MG-63, reduced the overall mRNAs level of PLCs, with special regard to some isoforms, with the notable exception of PLCB3. The partial replacement of the RPMI culture medium with supernatant obtained from cultures of 143B or MG-63 cells did not modify the expression of PLCB3. Furthermore, PLCB3 expression at the mRNA level increased partially and substituted RPMI with supernatant from HS888 cultures. This observation might accord to previous literature data indicating that the activity of PLC $\beta 3$ enzyme is essential to promote macrophage survival [40]. PLC $\beta 3$ is expressed in several tissues [10] and constitutes the upstream target of protein kinase $\mathrm{C} \varepsilon$, involved in the inflammatory response [41]. Recently, PLCB3 silencing was demonstrated to potentiate the Toll-like receptor inflammatory signaling cascade in cystic fibrosis bronchial epithelial cells that induce IL-8 release [42]. Along with morphological changes, indicating macrophage transition, THP-1 cells cultured in the presence of OS supernatants did not transcribe the mRNAs of PLC genes, depending on the OS cell line. In THP-1 cultures, the supernatant from 143B cell lines reduced the expression at the mRNA level of all PLC isoforms; while PLCD4 and PLCE were not expressed (Tables 1, 4, and 5).

Notably, THP-1 cells, which constitutively express PLC 84 and PLCE, did not transcribe the corresponding genes when cultured with OS supernatant (Table 1). In our previous work, we described that macrophages, including M0, M1, and M2, did not express PLCD4 and PLCE, both in a physiological state and after inflammatory stimuli [12](Table 4). PLC 84 is expressed in response to mitogenic stimulation and plays an important role in cell growth and tumorigenesis. PLC $\varepsilon$, a peculiar PLC isoform is involved in several signaling pathways, such as Ras and Rho signaling [43]. The administration of OS supernatants to THP1 cultures seemed to block the expression at the mRNA level of both PLCD4 and PLCE. That might suggest that OS supernatants, as inducing
THP-1 to differentiate into macrophages, modify the expression panel of PLCs at the mRNA level, which mutually changes. The different expressions of PLCD4 and PLCE might represent a further marker to discriminate the differentiation into macrophages.

The differences of PLCs expression at the mRNA level induced by adding OS supernatants to the cultures of THP-1 cells resulted in a statistically significant difference for PLCB1 and PLCG2 genes, which codify isoforms belonging to the so-called primary PLCs. PLC $\beta 1$ enzyme was demonstrated to be involved in myocyte [44] and adipocyte differentiation [11]. Moreover, PLC $\beta 1$ is related to Cyclin D3, which was described to control important signaling pathways in macrophages [45]. PLC $\gamma 2$ is highly expressed in hematopoietic lineage cells and plays a crucial role in immune responses [46, 47]. Mutations in the PLCG2 gene lead to dominantly inherited autoinflammatory disease with immunodeficiency $[48,49]$. In conclusion our results indicated that supernatants from cultures of OS cell lines contain cytokines/chemokines, which differ depending on the OS cell type. OS supernatants can induce the differentiation of THP-1 cells into macrophages. THP-1 cells cultured in OS supernatants express different panels of PLC enzymes. The expression panel of PLC enzymes differs during the differentiation of monocyte/ macrophage lineage THP-1 cells. Further studies are required to elucidate the role of PLC $\delta 4$ and PLC $\varepsilon$ both in the differentiation and in the immune system cells. More pieces of evidence might help to confirm whether the absence of these two PLC enzymes might be used as markers of macrophage differentiation. Selected PLC enzymes are involved in the signal transduction pathways; inducing the production of specific ILs. Inconsistent data are available concerning the effect of ILs on the expression of PLC enzymes. The role of the cytokines in the OS microenvironment, their effect upon tumor growth and spreading, as well as the possible relationships among selected cytokines and PI signal transduction pathway, deserve further observations.

\section{ACKNOWLEDGMENTS}

Not applicable 


\section{CONFLICT OF INTEREST}

The authors declared no conflict of interest.

\section{ETHICS APPROVAL}

Not applicable.

\section{REFERENCES}

1. Chen J, Rhodes TD, Hu WS. Comparison of the genetic recombination rates of human immunodeficiency virus type 1 in macrophages and T cells. J Virol. 2005;79(14):9337-40. DOI: 10.1128/JVI.79.14.93379340.2005 PMID: 15994830.

2. Mirabello L, Troisi RJ, Savage SA. International osteosarcoma incidence patterns in children and adolescents, middle ages and elderly persons. Int J Cancer. 2009;125(1):229-34 DOI: 10.1002/ijc.24320 PMID: 19330840.

3. Lo Vasco VR, Leopizzi M, Chiappetta C, Puggioni C, Di Cristofano C, Della Rocca C. Expression of phosphoinositide-specific phospholipase $\mathrm{C}$ enzymes in human skin fibroblasts. Connect Tissue Res. 2013;54(1):1-4. DOI: 10.3109/03008207.2012.712584 PMID: 22800439.

4. Llaverias G, Lacasa D, Vazquez-Carrera M, Sanchez RM, Laguna JC, Alegret M. Cholesterol regulation of genes involved in sterol trafficking in human THP-1 macrophages. Mol Cell Biochem. 2005;273(1-2):185-91. DOI: $10.1007 / \mathrm{s} 11010-005-0624-8$ PMID: 16013454.

5. Auwerx J, Sassone-Corsi P. AP-1 (Fos-Jun) regulation by IP-1: effect of signal transduction pathways and cell growth. Oncogene. 1992;7(11):2271-80. PMID: 1437149 .

6. Daigneault M, Preston JA, Marriott HM, Whyte MK, Dockrell DH. The identification of markers of macrophage differentiation in PMA-stimulated THP-1 cells and monocyte-derived macrophages. PLoS One. 2010;5(1):e8668. DOI: 10.1371/journal.pone.0008668 PMID: 20084270.

7. Genin M, Clement F, Fattaccioli A, Raes M, Michiels C. M1 and M2 macrophages derived from THP-1 cells differentially modulate the response of cancer cells to etoposide. BMC Cancer. 2015;15:577. DOI: 10.1186/s12885015-1546-9 PMID: 26253167.

8. Berges C, Naujokat C, Tinapp S, Wieczorek H, Hoh A, Sadeghi M, et al. A cell line model for the differentiation of human dendritic cells. Biochem Biophys Res Commun. 2005;333(3):896-907. DOI: 10.1016/j.bbrc.2005.05.171 PMID: 15963458 .

9. Xi YH, Li HZ, Zhang WH, Wang LN, Zhang L, Lin Y, et al. The functional expression of calcium-sensing receptor in the differentiated THP-1 cells. Mol Cell Biochem. 2010;342(1-2):233-40. DOI: 10.1007/s11010-010-04893 PMID: 20473557.

10. Suh PG, Park JI, Manzoli L, Cocco L, Peak JC, Katan M, et al. Multiple roles of phosphoinositide-specific phospholipase C isozymes. BMB Rep. 2008;41(6):415-34. DOI: 10.5483/bmbrep.2008.41.6.415 PMID: 18593525.

11. O'Carroll SJ, Mitchell MD, Faenza I, Cocco L, Gilmour
RS. Nuclear PLCbeta1 is required for 3T3-L1 adipocyte differentiation and regulates expression of the cyclin D3cdk4 complex. Cell Signal. 2009;21(6):926-35. DOI: 10.1016/j.cellsig.2009.02.002 PMID: 19385066.

12. Di Raimo T, Leopizzi M, Mangino G, Rocca CD, Businaro R, Longo L, et al. Different expression and subcellular localization of Phosphoinositide-specific Phospholipase C enzymes in differently polarized macrophages. J Cell Commun Signal. 2016;10(4):283-93. DOI: 10.1007/ s12079-016-0335-9 PMID: 27394153.

13. Lo Vasco VR. Phosphoinositide pathway and the signal transduction network in neural development. Neurosci Bull. 2012;28(6):789-800. DOI: 10.1007/s12264-0121283-x PMID: 23152330.

14. Lo Vasco VR, Cardinale G, Polonia P. Deletion of PLCB1 gene in schizophrenia-affected patients. J Cell Mol Med. 2012;16(4):844-51. DOI: 10.1111/j.15824934.2011.01363.x PMID: 22507702.

15. Bunney TD, Katan M. PLC regulation: emerging pictures for molecular mechanisms. Trends Biochem Sci. 2011;36(2):88-96. DOI: 10.1016/j.tibs.2010.08.003 PMID: 20870410.

16. Lo Vasco VR. Role of phosphoinositide-specific phospholipase $\mathrm{C}$ eta2 in isolated and syndromic mental retardation. Eur Neurol. 2011;65(5):264-9. DOI: 10.1159/000327307 PMID: 21474938.

17. Lo Vasco VR, Leopizzi M, Chiappetta C, Puggioni C, Di Cristofano C, Della Rocca C. Expression of Phosphoinositide-specific phospholipase $\mathrm{C}$ enzymes in human osteosarcoma cell lines. J Cell Commun Signal. 2013;7(2):141-50. DOI: 10.1007/s12079-013-0194-6 PMID: 23430511.

18. Lo Vasco VR, Longo L, Polonia P. Phosphoinositidespecific Phospholipase $\mathrm{C}$ betal gene deletion in bipolar disorder affected patient. J Cell Commun Signal. 2013;7(1):25-9. DOI: 10.1007/s12079-012-0182-2 PMID: 23161413.

19. Lo Vasco VR, Fabrizi C, Artico M, Cocco L, Billi AM, Fumagalli L, et al. Expression of phosphoinositide-specific phospholipase $\mathrm{C}$ isoenzymes in cultured astrocytes. J Cell Biochem. 2007;100(4):952-9. DOI: 10.1002/ jcb.21048 PMID: 17063484.

20. Lo Vasco VR, Leopizzi M, Puggioni C, Della Rocca C. Ezrin silencing remodulates the expression of Phosphoinositide-specific Phospholipase C enzymes in human osteosarcoma cell lines. J Cell Commun Signal. 2014;8(3):219-29. DOI: 10.1007/s12079-014-0235-9 PMID: 25073508 .

21. Lo Vasco VR, Leopizzi M, Puggioni C, Della Rocca C, Businaro R. Neuropeptide $\mathrm{Y}$ reduces the expression of PLCB2, PLCD1 and selected PLC genes in cultured human endothelial cells. Mol Cell Biochem. 2014;394(12):43-52. DOI: 10.1007/s11010-014-2079-2 PMID: 24903829.

22. Lo Vasco VR, Fabrizi C, Fumagalli L, Cocco L. Expression of phosphoinositide-specific phospholipase $\mathrm{C}$ isoenzymes in cultured astrocytes activated after 
stimulation with lipopolysaccharide. J Cell Biochem. 2010;109(5):1006-12. DOI: 10.1002/jcb.22480 PMID: 20082315.

23. Lo Vasco VR, Leopizzi M, d'abusco AS, Rocca CD. Comparison of Phosphoinositide-Specific Phospholipase C Expression Panels of Human Osteoblasts Versus MG63 and Saos Osteoblast-Like Cells. Avicenna J Med Biochem. 2016;4(2):6-34104. DOI: 10.17795/ajmb-34104.

24. Lo Vasco VR, Leopizzi M, Di Maio V, Della Rocca C. U-73122 reduces the cell growth in cultured MG-63 ostesarcoma cell line involving Phosphoinositide-specific Phospholipases C. Springerplus. 2016;5:156. DOI: 10.1186/s40064-016-1768-6 PMID: 27026853.

25. Lo Vasco VR, Leopizzi M, Di Maio V, Di Raimo T, Cesa S, Masci A, et al. LPS, Oleuropein and Blueberry extracts affect the survival, morphology and Phosphoinositide signalling in stimulated human endothelial cells. J Cell Commun Signal. 2017;11(4):317-27. DOI: 10.1007/ s12079-017-0391-9 PMID: 28452007.

26. Fais P, Leopizzi M, Di Maio V, Longo L, Della Rocca C, Tagliaro F, et al. Phosphoinositide-specific phospholipase $\mathrm{C}$ in normal human liver and in alcohol abuse. J Cell Biochem. 2018. DOI: 10.1002/jcb.28067 PMID: 30426534.

27. Lo Vasco VR. 1p36.32 rearrangements and the role of PI-PLC eta2 in nervous tumours. J Neurooncol. 2011;103(3):409-16. DOI: 10.1007/s11060-010-0422-3 PMID: 20878447.

28. Lo Vasco VR, Leopizzi M, Della Rocca C, Fais P, Montisci $\mathrm{M}$, Cecchetto $\mathrm{G}$. Impairment and reorganization of the phosphoinositide-specific phospholipase $\mathrm{C}$ enzymes in suicide brains. J Affect Disord. 2015;174:324-8. DOI: 10.1016/j.jad.2014.12.006 PMID: 25532079.

29. Lo Vasco VR, Calabrese G, Manzoli L, Palka G, Spadano A, Morizio E, et al. Inositide-specific phospholipase c betal gene deletion in the progression of myelodysplastic syndrome to acute myeloid leukemia. Leukemia. 2004;18(6):1122-6. DOI: 10.1038/sj.leu.2403368 PMID: $\underline{15085153}$

30. Lo Vasco VR, Leopizzi M, Puggioni C, Della Rocca C, Businaro R. Fibroblast growth factor acts upon the transcription of phospholipase $\mathrm{C}$ genes in human umbilical vein endothelial cells. Mol Cell Biochem. 2014;388(12):51-9. DOI: 10.1007/s11010-013-1898-X PMID: 24242047.

31. Chien HC, Chan PC, Tu CC, Day YJ, Hung LM, Juan CC, et al. Importance of PLC-Dependent PI3K/AKT and AMPK Signaling in RANTES/CCR5 Mediated Macrophage Chemotaxis. Chin J Physiol. 2018;61(5):266-79. DOI: 10.4077/CJP.2018.BAG584 PMID: 30384400.

32. Barthes J, Ozcelik H, Hindie M, Ndreu-Halili A, Hasan A, Vrana NE. Cell microenvironment engineering and monitoring for tissue engineering and regenerative medicine: the recent advances. Biomed Res Int. 2014;2014:921905. DOI: 10.1155/2014/921905 PMID: 25143954.

33. Wu TH, Li YY, Wu TL, Chang JW, Chou WC, Hsieh LL, et al. Culture supernatants of different colon cancer cell lines induce specific phenotype switching and functional alter- ation of THP-1 cells. Cell Immunol. 2014;290(1):107-15. DOI: 10.1016/j.cellimm.2014.05.015 PMID: 24960291.

34. Garimella R, Washington L, Isaacson J, Vallejo J, Spence M, Tawfik O, et al. Extracellular Membrane Vesicles Derived from 143B Osteosarcoma Cells Contain Pro-Osteoclastogenic Cargo: A Novel Communication Mechanism in Osteosarcoma Bone Microenvironment. Transl Oncol. 2014;7(3):331-40. DOI: 10.1016/j.tranon.2014.04.011 PMID: 25180057.

35. Fukayama S, Tashjian AH, Jr. Stimulation by parathyroid hormone of $45 \mathrm{Ca} 2+$ uptake in osteoblast-like cells: possible involvement of alkaline phosphatase. Endocrinology. 1990;126(4):1941-9. DOI: 10.1210/endo-126-4-1941 PMID: 2318151.

36. Kaminski M, Masaoka M, Karbowski M, Kedzior J, Nishizawa Y, Usukura J, et al. Ultrastructural basis for the transition of cell death mode from apoptosis to necrosis in menadione-treated osteosarcoma 143B cells. J Electron Microsc (Tokyo). 2003;52(3):313-25. DOI: 10.1093/ jmicro/52.3.313 PMID: 12892222.

37. Umare V, Pradhan V, Nadkar M, Rajadhyaksha A, Patwardhan M, Ghosh KK, et al. Effect of proinflammatory cytokines (IL-6, TNF-alpha, and IL-1beta) on clinical manifestations in Indian SLE patients. Mediators Inflamm. 2014;2014:385297. DOI: 10.1155/2014/385297 PMID: 25548434.

38. Goepfert A, Lehmann S, Wirth E, Rondeau JM. The human IL-17A/F heterodimer: a two-faced cytokine with unique receptor recognition properties. Sci Rep. 2017;7(1):8906. DOI: 10.1038/s41598-017-08360-9 PMID: 28827714.

39. Akdis M, Burgler S, Crameri R, Eiwegger T, Fujita H, Gomez E, et al. Interleukins, from 1 to 37, and interferon-gamma: receptors, functions, and roles in diseases. J Allergy Clin Immunol. 2011;127(3):701-21 e1-70. DOI: 10.1016/j.jaci.2010.11.050 PMID: 21377040.

40. Wang Z, Liu B, Wang P, Dong X, Fernandez-Hernando C, Li Z, et al. Phospholipase C beta3 deficiency leads to macrophage hypersensitivity to apoptotic induction and reduction of atherosclerosis in mice. J Clin Invest. 2008;118(1):195-204. DOI: 10.1172/JCI33139 PMID: 18079968 .

41. Zhong M, Murtazina DA, Phillips J, Ku CY, Sanborn BM. Multiple signals regulate phospholipase CBeta3 in human myometrial cells. Biol Reprod. 2008;78(6):1007-17. DOI: 10.1095/biolreprod.107.064485 PMID: 18322273.

42. Rimessi A, Bezzerri V, Salvatori F, Tamanini A, Nigro F, Dechecchi MC, et al. PLCB3 Loss of Function Reduces Pseudomonas aeruginosa-Dependent IL-8 Release in Cystic Fibrosis. Am J Respir Cell Mol Biol. 2018;59(4):428-36. DOI: 10.1165/rcmb.2017-0267OC PMID: 29668297.

43. Wang X, Fan Y, Du Z, Fan J, Hao Y, Wang J, et al. Knockdown of Phospholipase Cepsilon (PLCepsilon) Inhibits Cell Proliferation via Phosphatase and Tensin Homolog Deleted on Chromosome 10 (PTEN)/AKT Signaling Pathway in Human Prostate Cancer. Med Sci Monit. 
2018;24:254-63. DOI: 10.12659/msm.908109 PMID: 29330357.

44. Faenza I, Bavelloni A, Fiume R, Santi P, Martelli AM, Maria Billi A, et al. Expression of phospholipase $\mathrm{C}$ beta family isoenzymes in $\mathrm{C} 2 \mathrm{C} 12$ myoblasts during terminal differentiation. J Cell Physiol. 2004;200(2):291-6. DOI: 10.1002/jcp.20001 PMID: 15174099.

45. Ruiz A, Pauls E, Badia R, Torres-Torronteras J, Riveira-Munoz E, Clotet B, et al. Cyclin D3-dependent control of the dNTP pool and HIV-1 replication in human macrophages. Cell Cycle. 2015;14(11):1657-65. DOI: 10.1080/15384101.2015.1030558 PMID: 25927932.

46. Homma Y, Takenawa T, Emori Y, Sorimachi H, Suzuki K. Tissue- and cell type-specific expression of mRNAs for four types of inositol phospholipid-specific phospholipase C. Biochem Biophys Res Commun. 1989;164(1):406-12.
DOI: 10.1016/0006-291x(89)91734-8 PMID: 2553017.

47. Kurosaki T, Okada T. Regulation of phospholipase Cgamma2 and phosphoinositide 3-kinase pathways by adaptor proteins in B lymphocytes. Int Rev Immunol. 2001;20(6):697-711. DOI: 10.3109/08830180109045586 PMID: 11913946.

48. Zhou Q, Lee GS, Brady J, Datta S, Katan M, Sheikh A, et al. A hypermorphic missense mutation in PLCG2, encoding phospholipase Cgamma2, causes a dominantly inherited autoinflammatory disease with immunodeficiency. Am J Hum Genet. 2012;91(4):713-20. DOI: 10.1016/j. ajhg.2012.08.006 PMID: 23000145.

49. Comer FI, Parent CA. Phosphoinositides specify polarity during epithelial organ development. Cell. 2007;128(2):239-40. DOI: 10.1016/j.cell.2007.01.010 PMID: 17254962. 